\title{
MODELING OF THE CURRENT DENSITY DISTRIBUTION UNDER SURFACE POSTER-TIBIAL-NERVE ELECTRIC STIMULATOR Y.Hu* $^{\dagger}$, J.Yu*, K.D.K. Luk ${ }^{\dagger}$, Y.W.Wong ${ }^{\dagger}$, W.W.Lu', J.C.Y. Leong ${ }^{\dagger}$ \\ * Department of Biomedical Engineering, Tianjin University, Tianjin, 300072,P.R.C ${ }^{\dagger}$ Department of Orthopaedic Surgery, The University of Hong Kong, Hong Kong, P.R.C *E-mail: yhud@hkusua.hku.hk
}

\begin{abstract}
Stimulation of the posterior tibial nerve is commonly used in the measurement of somatosensory evoked potential (SEP). To improve the efficiency of stimulation, the potential field and current density distributions under the surface electrodes were modeled and simulated. In our model, three layers were assumed: (1) the air environment, (2) electrode and paste (3) human body (skin and soft tissues). The mirror method was used to analyze the potential field of point charge. Integration of the field and the area of the stimulus gave the potential field of one surface electric pole. The potential field distribution of the bipolar stimulator was obtained by superimposition of two unipolar's fields. Finally, the current density distribution was calculated by Laplace equation. The analytical solution of the potential field was found and the numerical solution of the current density distribution calculated. The potential field and current density distributions were simulated by 2-D plot. From the model and simulation, the potential and current density distributions were not found to be uniform under transcutaneous stimulation electrode and the maximum current density is located under the poles. We recommend that bipolar stimulator should be applied axially along the stimulated nerve course.

Keywords: current density distribution, potential field distribution, posterior tibial nerve
\end{abstract}

\section{Introduction}

Transcutaneous Electrical Nerve Stimulation (TENS) has been employed in many situations including nerve function stimulation and neural function detection [1]. Stimulation on the posterior tibial nerve for measurement of somatosensory evoked potential (SEP) is one of the applications [2].

SEP is the electrical response of the brain to an applied somatosensory stimulus, which is usually applied to the posterior tibial nerve. SEP has been carried out clinically for nearly 30 years, and has recently become one of the techniques for intra-operative spinal cord monitoring [3].

There are many factors that can influence the SEP output, such as temperature, blood pressure, anaesthesia and stimulus intensity. The design of the stimulator and the stimulation setup also play an important part in SEP measurement [4].

Study of the potential and current density distributions under TENS have become a challenge to many investigators. A number of mathematical models have been used to estimate potential and current distributions, using finite element method (FEM) [5-8]. Conductivity (linear or nonlinear) characteristics are described in the electrode-skin structure [6-7], but the dielectric properties of the field are not considered in the FEM approach.

To get the optimum efficiency and to reduce the overflow to non-target tissues, the current density distributions under the surface posterior tibial nerve stimuli were modeled and simulated in this study.

\section{Methods}

As fig. 1 shows, the electrode was applied over the posterior tibial nerve behind the medial malleoli. In this situation, the transversal section view was supposed as plane shape.

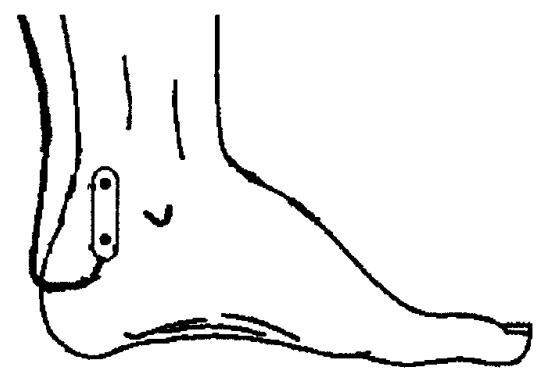

Fig.1 Application of surface bipolar stimuli over posterior tibial nerve

In our model, three layers were assumed for the stimulation. They are (1) the air environment with the electrode and jelly, (2) the human body (skin and soft tissues), (3). Nerve fiber. These are shown in fig. 2.

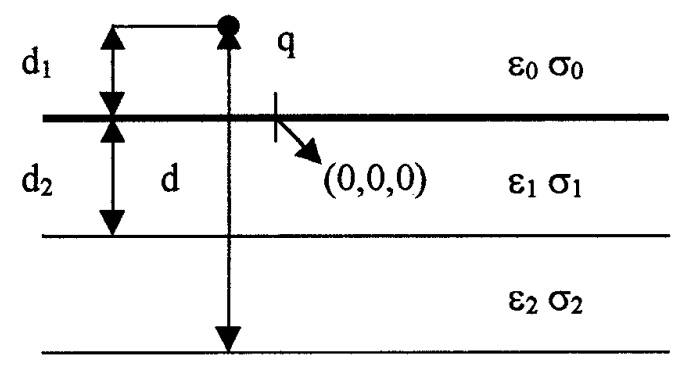

Fig.2 Three layers model of posterior tibial never stimulation

The frequency of stimulation used in the model was set at $5 \mathrm{~Hz}$, a similar value as clinic test. The mirror method was used to analyze the potential field and the current distribution. 
According to the Maxwell equation (ignoring the influence of the magnetic field), the potential field is given by the Laplace equation:

$$
\nabla^{2} \Phi=0
$$

Where $\Phi$ is the potential field in [V]. The electric field intensity and the current density are given by:

$$
\begin{aligned}
& E=-\nabla \phi \\
& \vec{J}=\sigma \vec{E}
\end{aligned}
$$

Where $E$ is the electric field intensity in $[\mathrm{V} / \mathrm{m}], \mathrm{J}$ is the current density in $\left[\mathrm{A} / \mathrm{m}^{2}\right]$ and $\sigma$ is the material conductivity in [S/m].

To analyze the charge field distribution, the point charge and its mirror charges were introduced. Thus the potential field of point charge was obtained. According to the associative property, the potential field under the stimulus is given by the integration of the stimulation current density and the area of the surface electrode. Therefore, the current density distribution can be calculated using equation (2).

Results

Following analysis by the above method, the potential field distributions are described by:

$$
\begin{aligned}
\phi_{0}= & \frac{q}{4 \pi \varepsilon_{1}} \cdot \frac{1}{\sqrt{x^{2}+y^{2}+\left(z-d_{1}\right)^{2}}} \\
& +\frac{q}{4 \pi \varepsilon_{1}} \sum_{k=0}^{\infty}\left[\frac{A^{k} B^{k+1}}{\sqrt{x^{2}+y^{2}+\left(z-2 k d-d_{1}\right)^{2}}}\right. \\
& +\frac{A^{k+1} B^{k}}{\sqrt{x^{2}+y^{2}+\left[\left(z-(2 k+1) d-d_{2}\right]^{2}\right.}} \\
& +\frac{A^{k+1} B^{k+1}}{\sqrt{x^{2}+y^{2}+\left[\left(z-(2 k+1) d-d_{2}\right]^{2}\right.}} \\
& \left.+\frac{q}{4 \pi \varepsilon_{1}} \cdot \frac{A^{k+1} B^{k+1}}{\sqrt{x^{2}+y^{2}+\left[\left(z-2(k+1) d-d_{1}\right]^{2}\right.}}\right] \\
& +\frac{q}{4 \pi \varepsilon_{1} \sum_{k=0}^{\infty}\left[\frac{1}{\sqrt{x^{2}+y^{2}+\left(z+2 k d+d_{1}\right)^{2}}}\right.} \\
& +\frac{A_{1}^{k+1} B^{k}}{\sqrt{x^{2}+y^{2}+\left[\left(z-(2 k+1) d-d_{2}\right]^{2}\right.}} \\
& +\frac{A^{k+1} B^{k+1}}{\sqrt{x^{2}+y^{2}+\left[\left(z+(2 k+1) d+d_{2}\right]^{2}\right.}}
\end{aligned}
$$

$$
\begin{aligned}
\phi_{1}= & \frac{q}{4 \pi \varepsilon_{1}} \cdot \frac{1}{\sqrt{x^{2}+y^{2}+\left(z-d_{1}\right)^{2}}} \\
& +\frac{q}{4 \pi \varepsilon_{1}} \sum_{k=0}^{\infty}\left[\frac{A^{k} B^{k+1}}{\sqrt{x^{2}+y^{2}+\left(z-2 k d-d_{1}\right)^{2}}}\right. \\
& +\frac{A^{k+1} B^{k}}{\sqrt{x^{2}+y^{2}+\left[\left(z-(2 k+1) d-d_{2}\right]^{2}\right.}} \\
& +\frac{A^{k+1} B^{k+1}}{\sqrt{x^{2}+y^{2}+\left[\left(z-(2 k+1) d-d_{2}\right]^{2}\right.}} \\
& \left.+\frac{A^{k+1} B^{k+1}}{\sqrt{x^{2}+y^{2}+\left[\left(z-2(k+1) d-d_{2}\right]^{2}\right.}}\right]
\end{aligned}
$$

Where $A=\frac{\sigma_{1}-\sigma_{2}}{\sigma_{1}+\sigma_{2}}, \quad B=\frac{\sigma_{1}-\sigma_{0}}{\sigma_{1}+\sigma_{0}}$

In each zone the associated potential field satisfies the appropriate boundary conditions as below:

$$
\begin{aligned}
& \left.\phi_{0}\right|_{z \rightarrow 0}=\left.\left.\phi_{1}\right|_{z \rightarrow 0} \quad \phi_{1}\right|_{z \rightarrow d}=\left.\phi_{2}\right|_{z \rightarrow d} \\
& \left.\varepsilon_{0} \frac{\phi_{0}}{z}\right|_{z \rightarrow 0}=\left.\left.\varepsilon_{1} \frac{\phi_{1}}{z}\right|_{z \rightarrow 0} \quad \varepsilon_{1} \frac{\phi_{1}}{z}\right|_{z \rightarrow d}=\left.\varepsilon_{2} \frac{\phi_{2}}{z}\right|_{z \rightarrow d}
\end{aligned}
$$

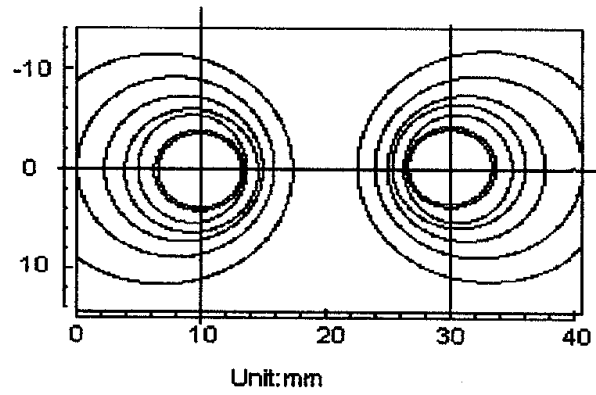

(a)

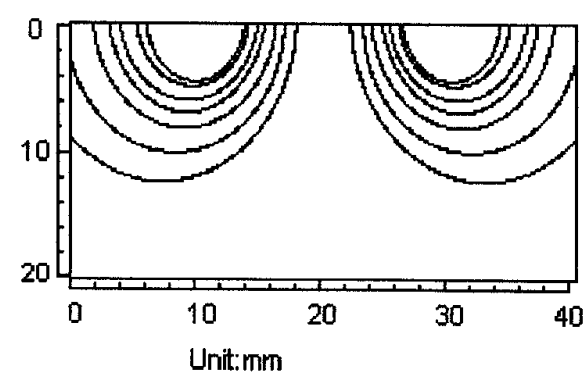

(b)

Fig. 3 Field distribution under surface electrode

(a) shows the surface of skin, two intersection points indicate the bipolar electrode.

(b) shows the cross section view of the potential field distribution under the bipolar electrode. 
Fig. 3 shows the potential field distribution under the surface electrode.

The numerical solution was further calculated to simulate the current density distribution by using equation (2). The results are shown in Fig.4.

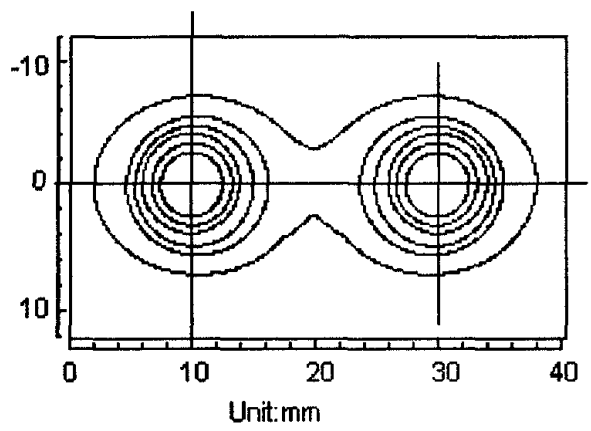

(a)

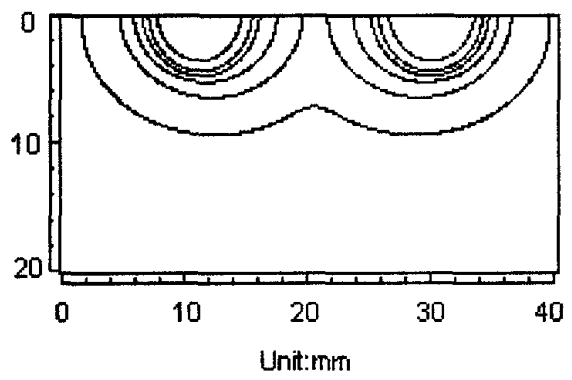

(b)

Fig.4 Current density distribution of the transcutaneous electric stimulation over the posterior tibial nerve

(a) shows the current density distribution on the surface of skin, two intersection points indicate the bipolar electrode.

(b) shows the cross section view of the current density distribution under the bipolar electrode.

The above two figures show that the distributions of current density under the transcutaneous posterior tibial nerve electric stimulation is different from the distribution of the potential field.

In fig.3, the potential distribution shows that the highest potential locate under the electrode, while the potential is zero in the middle area of the field. The current density distribution shows that the current density in the middle area of the field is not zero. It is $20 \mathrm{~dB}$ weaker than the stimulation current density. In fig.4(b), the distribution indicates that only $10 \%$ of the surface stimulation current density could arrive $10 \mathrm{~mm}$ deep in the body, which is approximate the nerve fiber's level.

Discussion and Conclusion

The potential and the current density distributions were calculated by the analytical solution and the numerical solution. A complete analytical solution would be more accurate, but is difficult to obtain. The accuracy of the numerical solution is adequate given variations in the field and current distributions of the model. The results showed the potential and current fields under the stimuli were not uniform. It is found that the maximum current density was located under the poles. The longer the distance from the stimulus location to the stimulating point was, the weaker the current density introduced. In other words, the stimulation bipolar should be applied along the stimulated nerve course.

Acknowledgement

This study was supported by the Research Grants Council (\#227/95M) from University of Hong Kong and the Tianjin 21 Century Fund from Tianjin Science and Technology Council.

\section{Reference:}

[1] Ferdjallah M, Bostick FX Jr, Barr RE: Potential and current density distributions of cranial electrotherapy stimulation (CES) in a four-concentric spheres model. IEEE Trans Biomed Eng 1996 Sep;43(9):939-943

[2] Nathan SS, Sinha SR, Gordon B, Lesser RP, Thakor NV: Detection of current density distribution generated by electrical stimulation of the human cerebral cortex. Electroencephalogr Clin Neurophysiol 1993;86:183-192

[3] Nash-CL Jr, Lorig-RA, Schatzinger-LA, Brown-RH: Spinal cord monitoring during operative treatment of the spine. Clinical Orthopaedics and Related research,1977, 126:100-105

[4] Nuwer-MR, Dawson-EG, Carlson-LG, et. al. : SEP spinal cord monitoring reduces neurologic deficits after scoliosis surgery: results of a large multicenter survey. Electroencephalogr-Clin-Neurophysiol. 1995; 96(1): 6-11 [5] Rijkhoff NJ, Holsheimer J, Koldewijn EL, et.al.: Selective stimulation of sacral nerve roots for bladder control: a study by computer modeling.IEEE Trans Biomed Eng 1994 May;41(5):413-424

[6] Voo K, Kumaresan S, Pintar FA, Yoganandan N, Sances A Jr: Finite-element models of the human head. Med Biol Eng Comput 1996 Sep;34(5):375-381

[7] Panescu D, Webster JG, Stratbucker RA: A nonlinear finite element model of the electrode-electrolyte-skin system. IEEE Trans Biomed Eng 1994 Jul;41(7):681-687

[8] Panescu D, Webster JG, Stratbucker RA: A nonlinear electrical-thermal model of the skin. IEEE Trans Biomed Eng 1994 Jul;41(7):672-680

[9] Rattay F: Analysis of models for extracellular fiber stimulation. IEEE Trans Biomed Eng 1989 Jul;36(7):676-682 